\title{
Caracterización de la microbiota intestinal en robalo (Centropomus sp) y aislamiento de bacterias probióticas potenciales
}

\section{Characterization of the intestinal microbiota in snook (Centropomus sp) and isolation of potential probiotic bacteria}

\author{
Adrian E. Zatán ${ }^{2,4}$, Deysy Castillo ${ }^{3}$, Arnaldo E. Castañeda ${ }^{1,2}$, \\ Manuel A. Feria ${ }^{1,2}$, Odalis E. Toledo ${ }^{1,2}$, Jorge L. Aguilar ${ }^{2,4}$, \\ Mario D. Cueva ${ }^{3}$, Emmerik Motte ${ }^{1}$
}

\section{Resumen}

El objetivo del estudio fue caracterizar la microbiota del tracto intestinal de robalo (Centropomus $\mathrm{sp}$ ), proveniente de medio natural y cautiverio, además de aislar e identificar bacterias con potencial probiótico. La microbiota intestinal se caracterizó mediante metagenómica dirigida al gen $16 \mathrm{~S}$ ARNr. Además, se sembraron muestras de mucus intestinal en medio de cultivo MRS para el aislamiento bacteriano. La identificación molecular se hizo mediante la secuenciación del gen 16S ARNr. Las capacidades probióticas se evidenciaron mediante ensayos proteolíticos y de antagonismo bacteriano frente a Plesiomonas shigelloides, Aeromonas hydrophila, Aeromonas veronii y Vibrio harveyi. Los géneros bacterianos con mayor abundancia fueron Clostridium, Photobacterium, Cetobacterium y Rubritepida. Las especies bacterianas, incluyendo Klebsiella sp, dos cepas de Weisiella cibaria y Lactococcus sp fueron aisladas en agar MRS a partir del tracto intestinal. Las cepas $W$. cibaria mostraron amplio espectro antagonista y actividad proteolítica positiva.

Palabras clave: Centropomus, microbiota, metagenómica, probióticos

\footnotetext{
${ }^{1}$ Incabiotec, Tumbes, Perú

${ }^{2}$ Pezbiotec, Tumbes, Perú

${ }^{3}$ Cooperativa de trabajadores Biotecoop, Tumbes, Perú

${ }^{4}$ Facultad de Ingeniería Pesquera y Ciencias del Mar, Universidad Nacional de Tumbes, Tumbes, Perú

${ }^{5}$ E-mail: pezbiotecsac@gmail.com
}

Financiamiento: Círculo de investigación en biotecnología molecular para el desarrollo y sostenibilidad del sector acuícola del Perú. Código: 132-2015 (Cienciactiva - Concytec)

Recibido: 3 de mayo de 2019

Aceptado para publicación: 16 de abril de 2020

Publicado: 11 de agosto de 2020 
The aim of this study was to characterize the microbiota of the intestinal tract of robalo (Centropomus sp), from natural environment and captivity, and to isolate and identify bacteria with probiotic potential. The intestinal microbiota was characterized by metagenomics directed to the $16 \mathrm{~S}$ rRNA gene. In addition, samples of intestinal mucus were cultured in MRS medium for bacterial isolation. Molecular identification was done by sequencing the $16 \mathrm{~S}$ rRNA gene. Probiotic capabilities were evidenced by proteolytic and bacterial antagonism assays against Plesiomonas shigelloides, Aeromonas hydrophila, Aeromonas veronii and Vibrio harveyi. The most abundant bacterial genera were Clostridium, Photobacterium, Cetobacterium and Rubritepida. Bacterial species, including Klebsiella sp, two strains of Weisiella cibaria and Lactococcus sp were isolated on MRS agar from the intestinal tract. W. cibaria strains showed broad antagonistic spectrum and positive proteolytic activity.

Key words: Centropomus, microbiota, metagenomics, probiotics

\section{INTRODUCCIÓN}

La producción acuícola mundial alcanzó 80 millones de toneladas (TM) de pescado en el año 2016, donde la maricultura representó el 35\% (FAO, 2018). A nivel nacional, la maricultura produjo el $40 \%$ de la producción acuícola total durante 2016-2017; sin embargo, la piscicultura aún se encuentra en proceso de desarrollo a pesar de su amplia variedad de especies (PRODUCE, 2017).

Un recurso hidrobiológico marino de importancia comercial en el norte del Perú es el Centropomus sp, conocido comúnmente como robalo, que se distribuye desde Baja California Sur en México hasta Paita en Perú (Muhlia et al., 1994). Es considerado un candidato potencial para su producción intensiva, debido a su rápida adaptabilidad y buena calidad de carne, convirtiéndose en una alternativa rentable (Cerqueira y Tsuzuki, 2009; Ramos y Palas, 2013); sin embargo, tiene como limitantes la dificultad de producir juveniles en cantidades suficientes y la alta mortandad en la etapa larvaria (Souza et al., 2010). Gran parte de esta mortandad es asociada principalmente a enfermedades infecciosas bacterianas, las cuales proliferan de- bido al aumento de las condiciones de estrés (Austin, 2005; Peso-Echarri et al., 2012; Lafferty et al., 2015).

Para contrarrestar las enfermedades infecciosas, los antibióticos se han convertido en el tratamiento profiláctico y terapéutico más utilizado (Romero et al., 2012); sin embargo, su uso indiscriminado a largo plazo selecciona bacterias resistentes a drogas (multidrogo-resistentes) perjudicando la salud pública (Banerjee y Ray, 2017), además de afectar microorganismos de la microbiota nativa del hospedero, conllevando a la proliferación de patógenos oportunistas (Modi et al., 2014).

La microbiota es un ecosistema microbiano dinámico y complejo, que se encuentra asociado a la piel, branquias y tracto gastrointestinal, habita en forma estable e interacciona entre sí, autorregulando su concentración numérica (Nayak, 2010; Merrifield y Rodiles, 2015). Como parte de la microbiota se encuentran bacterias benéficas que participan en la inmunidad del hospedero (Lazado y Caipang, 2014), siendo sus funciones más resaltantes: 1) modulación del sistema inmune, 2) suministración de nutrientes, 3) producción de compuestos antagónicos frente a 
patógenos, y 4) producción de enzimas extracelulares, como proteasas y lipasas (Pirarat et al., 2011; Drider et al., 2016; Zorriehzahra et al., 2016).

Para la correcta selección de probióticos se requieren técnicas moleculares de vanguardia como la secuenciación de nueva generación (NGS) (Cui et al., 2017) que permiten analizar la biodiversidad de las comunidades microbianas presentes, así como también, una mejor comprensión de la relación entre los aislados y la microbiota total (Ju y Zhang, 2015; Oulas et al., 2015). Por lo tanto, conociendo la microbiota intestinal de los peces se pueden descubrir probióticos potenciales que reduzcan la aparición de enfermedades infecciosas y el uso de antibióticos (Wang et al., 2008). El presente estudio tuvo como objetivo caracterizar, mediante metagenómica, la composición de la microbiota intestinal nativa del robalo Centropomus sp e identificar bacterias con potencial probiótico.

\section{Materiales y Métodos}

\section{Colección de la Muestra}

Tres robalos de estado juvenil y aparentemente sanos fueron colectados de los distritos de Zarumilla (medio natural), Corrales (medio natural) y Puerto Pizaro (en cautiverio), localizados en la región Tumbes, Perú, y fueron codificados como R1, R2 y R3, respectivamente. Se procedió a la eutanasia con previa anestesia con eugenol. Se diseccionaron en condiciones asépticas y se colectó $1 \mathrm{~g}$ por pez de intestino libre de contenido intestinal en tubos de $2 \mathrm{ml}$ con etanol absoluto. Finalmente, las muestras se trasladaron al laboratorio para estudios metagenómicos.

\section{Aislamiento Bacteriano \\ Se realizó un frotis intestinal utilizando hisopos estériles y se colocaron en tubos de $2 \mathrm{ml}$ con caldo Man Rogosa Sharpe (MRS). Las muestras se incubaron a temperatura}

ambiente durante $24 \mathrm{~h}$. Posteriormente se realizaron diluciones seriadas $\left(1: 10\right.$, de $10^{-1}$ a $\left.10^{-6}\right)$ y se sembraron por esparcimiento en placas Petri con agar MRS. Las colonias fueron subcultivadas por el método de agotamiento hasta obtener colonias puras.

\section{Antagonismo Bacteriano y Actividad Proteolítica}

Las bacterias aisladas se enfrentaron a cuatro bacterias patógenas de peces (Plesiomonas shigelloides, Aeromonas hydrophila, Aeromonas veronii y Vibrio harveyi) utilizando el método de difusión en agar con modificaciones (Balcázar et al., 2008). Las bacterias patógenas se cultivaron en caldo Triptic Soy Broth (TSB) durante $24 \mathrm{~h} \mathrm{a} 28^{\circ} \mathrm{C}$ y luego se sembraron $100 \mu 1$ de cada bacteria patógena en placas con Triptic Soy Agar (TSA) mediante el método de esparcimiento en superficie. Posteriormente, se realizaron pocillos en el agar con la ayuda de una pipeta Pasteur estéril y se agregaron $30 \mu 1\left(5 \times 10^{8}\right.$ unidades formadoras de colonia, UFC) de las bacterias aisladas. Finalmente, se incubaron a $28{ }^{\circ} \mathrm{C}$ durante $24 \mathrm{~h}$. Las zonas de actividad inhibitoria fueron calculadas en milímetros $(\mathrm{mm})$, considerándose positivas aquellas que mostraron una zona clara de inhibición.

La actividad proteolítica se evaluó en agar Skim Milk (5\%), método adaptado de Reda et al (2017). Se sembraron $30 \mu l$ de cada bacteria potencialmente probiótica en sus respectivos pocillos y se incubaron a temperatura ambiente durante $24 \mathrm{~h}$. Los aislados con actividades proteolíticas mostraron zonas transparentes alrededor.

\section{Extracción de ADN y PCR}

Se extrajo ADN metagenómico bacteriano a partir de $1 \mathrm{~g}$ de tejido intestinal utilizando el kit DNeasy PowerSoil ${ }^{\circledR}$ (Qiagen, Alemania), siguiendo las instrucciones del fabricante. Se extrajo ADN genómico bacteriano de los aislados utilizando el método CTAB (bromuro de hexadeciltrimetila- 


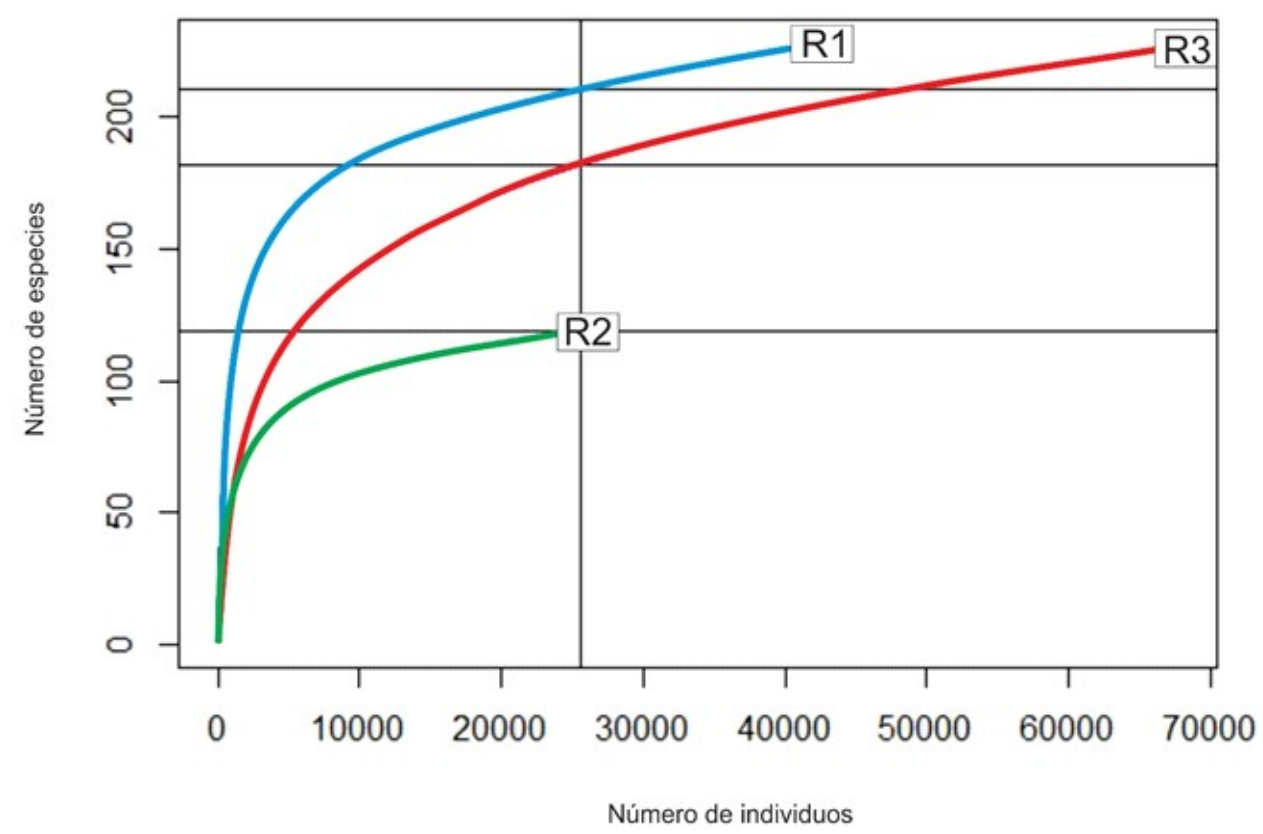

Figura 1. Curvas de rarefacción que muestran la diversidad de especies en las microbiotas intestinales de tres muestras de robalo (Centropomus sp) con relación al número de secuencias obtenidas

monio). La amplificación del gen 16S ARNr se hizo utilizando los cebadores universales 27F (5'-AGAGTTTAGTCMTG-GCTCAG3') y 1492R (5'-GGYTACCTT-GTTACGACTT-3').

La reacción en cadena de la polimerasa (PCR) se realizó mediante las siguientes condiciones: un ciclo a $94{ }^{\circ} \mathrm{C}$ durante $5 \mathrm{~min}$, seguido de 35 ciclos a $94{ }^{\circ} \mathrm{C}$ durante $30 \mathrm{~s}, 58^{\circ} \mathrm{C}$ durante $45 \mathrm{~s}, 7^{\circ} \mathrm{C}$ durante 1 min y $30 \mathrm{~s}$, y un ciclo final de $72{ }^{\circ} \mathrm{C}$ durante $6 \mathrm{~min}$. Se verificaron los productos de PCR en un gel al 1.5\% de agarosa teñido con bromuro de etidio.

\section{Secuenciación y Análisis}

Los productos amplificados y ADN metagenómico se enviaron a Macrogen (USA) y MRDNA (USA), respectivamente, para su secuenciación. Las secuencias obte- nidas fueron analizadas mediante los softwares MEGA 6 y Mothur.

\section{Resultados y Discusión}

La microbiota intestinal es un factor importante en la nutrición e inmunidad de los peces, por lo que su estudio es primordial, especialmente en especies de gran valor comercial. La metagenómica, parte de la genómica que estudia el ADN global de una comunidad de microorganismos, permite también la caracterización de bacterias con propiedades benéficas conocidas comúnmente como probióticos (Tarnecki et al., 2017).

En el presente estudio se caracterizó la microbiota intestinal mediante NGS dirigida al gen 16S ARNr de tres individuos de robalo (dos de medio natural y uno de cautiverio). 

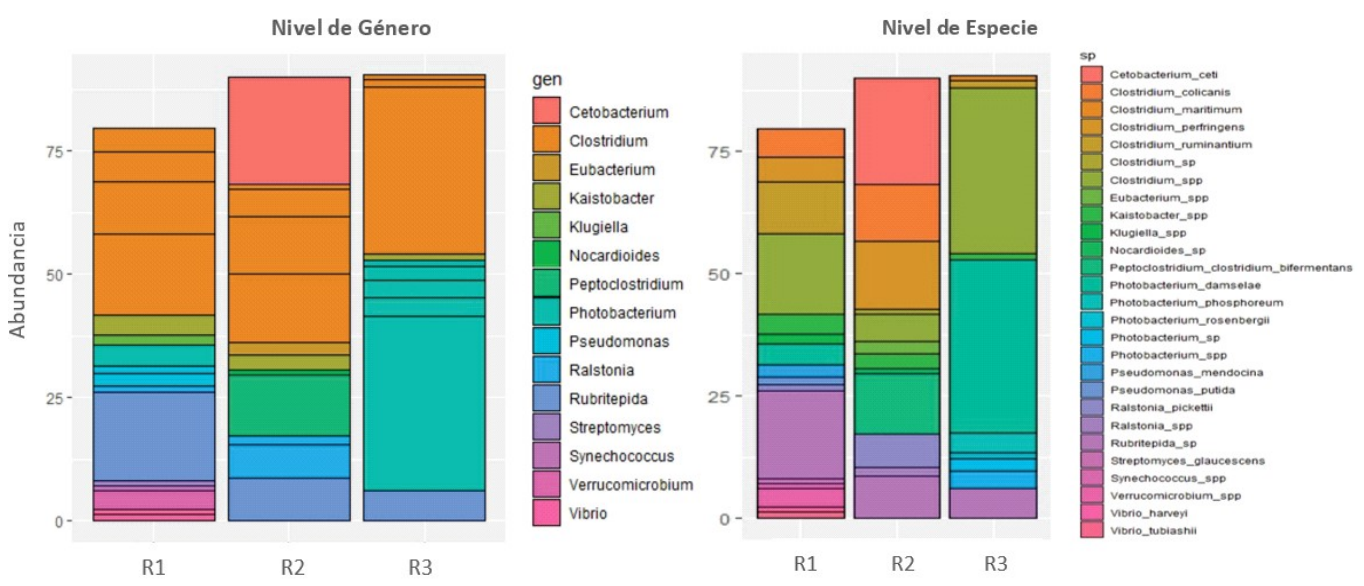

Figura 2. Composición taxonómica de la microbiota intestinal del robalo (Centropomus sp), Tumbes, Perú

La curva de rarefacción muestra el número de especies con relación al número de lecturas realizadas, donde las muestras R1 y R3 poseen una riqueza de alrededor de 220 especies y la muestra R2 de 120. La riqueza de especies estuvo relacionado al número de unidades taxonómicas operativas (OTUs, por sus siglas en inglés) establecido para cada muestra (Figura 1).

El estudio metagenómico de la muestra R1 mostró 11 géneros sobresalientes, de los cuales Rubritepida (17.94\%) y Clostridium (37.91\%) fueron los más dominantes. La muestra R2 presentó ocho géneros de los cuales resaltan Cetobacterium (12.44\%) y Clostridium (32.16\%). La muestra R3 presentó cuatro géneros sobresalientes en donde Photobacterium (49.24\%) y Clostridium (36.53\%) tuvieron mayor predominancia (Figura 2). Clostridium, del phylum Firmicutes, fue el género más dominante y estuvo presente en las tres muestras. Estudios recientes destacan que especies de los phylum Proteobacteria, Firmicutes, Actinobacteri, Fusobacteria, Bacteroidetes y Tenericutes son los más dominantes en el tracto gastrointestinal de los peces teleósteos (Llewellyn et al., 2014; do Vale Pereira et al., 2017).
Otros géneros sobresalientes fueron: Rubritepida, que requiere $\mathrm{NaCl}$ o sales marinas para su crecimiento. Es encontrado, además, en aguas termales saladas o lagos salinos. Este hallazgo tiene relación con el lugar de procedencia de las muestras, que son zonas estuarinas y de temperatura cálida. También se logró identificar Cetobacterium, que ha sido aislado a partir del intestino de peces como Oreochromis niloticus, Arapaima gigas, Cyprinus carpio y posee la capacidad de producir vitamina B12 (Ramírez et al., 2018), sugiriendo su rol en el metabolismo celular. Por otra parte, la abundancia relativa de especies de Clostridum en las muestras de robalo puede explicarse debido a su comportamiento simbionte con peces marinos hospederos (Clements et al., 2009), mientras que la presencia de Phtobacterium y Vibrio está relacionado al tipo de alimentación, y son encontrados comúnmente en peces carnívoros como Atlantic salmon, Salmo salar y Black rockcod, entre otros (Egerton et al., 2018).

Otros estudios mencionan que Photobacterium y Clostridium podrían ayudar a la digestión como también en la suplementación de ácidos grasos y vitaminas (Balcázar 
Cuadro 1. Actividad antagónica ${ }^{1}$ de aislados de intestino de robalo (Centropomus sp) frente a cuatro patógenos de peces (Tumbes, Perú)

\begin{tabular}{llllll}
\hline Código & $\begin{array}{l}\text { Identificación } \\
\text { molecular }\end{array}$ & $\begin{array}{l}\text { Plesiomonas } \\
\text { shigelloides }\end{array}$ & $\begin{array}{l}\text { Aeromonas } \\
\text { hidrophyla }\end{array}$ & $\begin{array}{l}\text { Aeromonas } \\
\text { veronii }\end{array}$ & $\begin{array}{l}\text { Vibrio } \\
\text { harveyi }\end{array}$ \\
\hline HE3B-3 & Klebsiella sp & ++ & +++ & +++ & +++ \\
HE2C-4 & Weisella cibaria & + & +++ & +++ & +++ \\
HE3C-4 & Weisella cibaria & ++ & +++ & +++ & +++ \\
HE2C-1 & Lactococcus sp & + & - & - & ++ \\
\hline
\end{tabular}

1 '_' No evidenció formación de halos, ' + ' Halos de 4-8 mm, '++' Halos de 8-12 mm, '+++' Halos $>12 \mathrm{~mm}$

et al., 2006; Ray et al., 2012). Más allá de la simbiosis natural, algunas especies de Clostridium han sido utilizadas como probióticos en Oncorhynchus mykiss, incrementando la resistencia a vibriosis (Sakai et al., 1995); sin embargo, este género también está conformado por especies patógenas como $C$. botulinum, frecuentemente asociado a enfermedades en peces marinos.

La identificación molecular de los aislados mostró tres especies, incluyendo Klebsiella sp, dos cepas de Weisiella cibaria y Lactococcus sp. El género Klebsiella es conocido por ser responsable de una variedad de enfermedades en peces como Labeo rohita y Amphiprion nigripes (Gopi et al., 2016; Das et al., 2018). Por otro lado, cepas identificadas como Lactococcus sp y Weissella cibaria han sido reportadas como probióticos de uso común en la acuicultura. Heo et al. (2013) demostraron la capacidad probiótica de L. lactis en Paralichthys olivaceus, debido a la activación del sistema inmune. Por otro lado, W. cibaria ha sido aislada a partir del intestino de Salmo trutta y Salmo salar indicando que es un componente común de la microbiota intestinal de peces marinos (Al-Hisnawi et al., 2014); sin embargo, también ha sido aislada del intestino de peces de agua dulce (Maji y Mohanty et al., 2017). Su capacidad probiótica ha sido demostrada al reducir el número de bacterias patógenas y estimular la presencia de bacterias ácido lácticas (BAL) en la microbiota intestinal de Pseudoplatystoma sp (Mouriño et al., 2011).

La capacidad antagónica de BAL aislados del intestino de peces frente a patógenos bacterianos ha sido ampliamente demostrada. Además, se menciona que es la propiedad más importante de una bacteria con potencial probiótico (Banerjee y Ray, 2017). En el presente estudio, los aislados HE3B-3 Klebsiella sp, HE2C-4 W. cibaria, HE3C-4 W. cibaria y HE2C-1 Lactococcus sp demostraron su capacidad antagónica frente al menos una cepa patógena (Cuadro 1). Esto puede deberse a las propiedades que poseen las BAL para producir sustancias bactericidas como bacteriocinas, peróxido de hidrógeno y proteasas, entre otros (Zorriehzahra et al., 2016). Finalmente, Klebsiella sp y $W$. cibaria fueron las cepas con mejores capacidades antagónicas; no obstante, Klebsiella es considerada comúnmente un patógeno oportunista (Austin, 2005), mientras que $W$. cibaria es considerada como probiótico. 
La microbiota cumple una función importante en la nutrición de los peces, y se debe principalmente a la producción de enzimas (Ray et al., 2012). En este estudio, los aislados HEB-3, HE2C-4, HE3C-4 y HE2C-1 fueron capaces de producir proteasas extracelulares demostradas en la formación de zonas transparentes alrededor de los pocillos en agar Skim Milk (5\%).

\section{Literatura Citada}

1. Al-Hisnawi A, Ringo E, Davies SJ, Waines P, Bradley G, Merriefield DL. 2014. First report on the autochthonus gut microbiota of brow trout (Salmo trutta Linnaeus). Aquac Res 46: 29622971. doi: 10.1111/are.12451

2. Austin B. 2005. Bacterial pathogens of marine fish. In: Oceans and health: pathogens in the marine environment. Boston, USA: Springer. p 391-413.

3. Balcázar JL, De Blas I, Ruíz-Zarzuela $I$, Cunningham $D$, Vendrell $D$, Muzquiz JL. 2006. The role of probiotics in aquaculture. Vet Microbiol 114: 173-186. doi: 10.1016/j.vetmic.2006.01.009

4. Balcázar JL, Vendrell D, de Blas I, Ruiz-Zarzuela I, Muzquiz JL, Girones O. 2008. Characterization of probiotic properties of lactic acid bacteria isolated from intestinal microbiota of fish. Aquaculture 278: 188-191. doi: 10.1016/ j.aquaculture.2008.03.014

5. Banerjee G, Ray AK. 2017. The advancement of probiotics research and its application in fish farming industries. Res Vet Sci 115: 66-77. doi: 10.1016/j.rvsc.2017.01.016

6. Cerqueira VR, Tsuzuki MY. 2009. A review of spawning induction, larviculture, and juvenile rearing of the fat snook, Centropomus parallelus. Fish Physiol Biochem 35: 17-28. doi: 10.1007/s10695008-9245-y
7. Clements KD, Raubenheimer D, Choat JH. 2009. Nutritional ecology of marine herbivorous fishes: ten years on. Funct Ecol 23: 79-92. doi: 10.1111/j.13652435.2008.01524.x

8. Cui J, Xiao M, Liu M, Wang Z, Liu F, Guo L, Huang S. 2017. Coupling metagenomics with cultivation to select host specific probiotic micro organisms for subtropical aquaculture. J Appl Microbiol 123: 1274-1285. doi: 10.1111/ jam. 13555

9. Das A, Acharya S, Behera BK, Paria P, Bhowmick S, Parida PK, Das BK. 2018. Isolation, identification and characterization of Klebsiella pneumoniae from infected farmed Indian major carp Labeo rohita (Hamilton 1822) in West Bengal, India. Aquaculture 482: 111-116. doi: 10.1016/j.aquaculture.2017.08.037

10. do Vale Pereira G, da Cunha DG, Pedreira-Mourino JL, Rodiles A, Jaramillo-Torres A, Merrifield DL. 2017. Characterization of microbiota in Arapaima gigas intestine and isolation of potential probiotic bacteria. J Appl Microbiol 123: 1298-1311. doi: 10.1111/ jam. 13572

11. Drider D, Bendali F, Naghmouchi K, Chikindas ML. 2016. Bacteriocins: not only antibacterial agents. Probiotics Antimicrob 8: 177-182. doi: 10.1007/s12602016-9223-0

12. Egerton S, Culloty S, Whooley J, Stanton C, Ross RP. 2018. The gut microbiota of marine fish. Front Microbiol 9: 873. doi: 10.3389/fmicb.2018.00873

13. [FAO] Organización de las Naciones Unidas para la Alimentación y la Agricultura. 2018. El estado mundial de la pesca y la acuicultura. Roma, Italia. [Internet]. Disponible en: http:// www.fao.org/3/i9540es/19540es.pdf

14. Gopi M, Kumar TTA, Prakash S. 2016. Opportunistic pathogen Klebsiella pneumoniae isolated from Maldive's 
clown fish Amphiprion nigripes with hemorrhages at Agatti Island, Lakshadweep archipelago. Int J Fisheries Aquatic Studies 4: 464-467.

15. Heo WS, Kim YR, Kim EY, Bai SC, Kong IS. 2013. Effect of dietary probiotic, Lactococcus lactis subsp. Lactis I2, supplementation on the growth and immune response of olive flounder (Paralichthys olivaceus). Aquaculture 376: 20-24. doi: 10.1016/j.aquaculture.2012.11.009

16. Ju F, Zhang T. 2015. 16S rRNA gene high-throughput sequencing data mining of microbial diversity and interactions. Appl Microbiol Biot 99: 4119-4129. doi: 10.1007/s00253-015-6536-y

17. Lafferty KD, Harvell CD, Conrad JM, Friedman CS, Kent ML, Kuris AM, Saksida SM. 2015. Infectious diseases affect marine fisheries and aquaculture economics. Annu Rev Mar Sci 7: 47196. doi: 10.1146/annurev-marine-010814015646

18. Lazado CC, Caipang CMA. 2014. Mucosal immunity and probiotics in fish. Fish Shellfish Immun 39: 78-89. doi: 10.1016/j.vetmic.2010.08.024

19. Llewellyn MS, Boutin S, Hoseinifar SH, Derome N. 2014. Teleost microbiomes: the state of the art in their characterization, manipulation and importance in aquaculture and fisheries. Front Microbiol 5: 207. doi: 10.3389/fmicb.2014.00207

20. Maji UJ, Mohhanty S. 2017. Genotypic characterization of lactic acid bacteria in gut microbiome of freshwater fish. Microbiology 86: 276-285. doi: 10.1134/ S0026261717020138

21. Merrifield DL, Rodiles A. 2015. The fish microbiome and its interactions with mucosal tissues. In: Mucosal health in aquaculture. Academic Press. p 273-295. doi: 10.1016/B978-0-12-417186-2.00010-8
22. Modi SR, Collins JJ, Relman DA. 2014. Antibiotics and the gut microbiota. J Clin Invest 124: 4212-4218. doi: 10.1172/ JCI72333

23. Mouriño JLP, De Nascimento Viera F, Jatoba AB, De Silva BC, Jesus GFA, Seiffert WQ, Martins ML. 2011. Effect of dietary supplementation of inulin and W. cibaria on haemato-immunological parameters of hybrid surubim (Pseudoplatystoma $\mathrm{sp}$ ). Aquacult Nutr 18: 7380. doi: 10.1111/j.1365-2095.2011.00879.x

24. Muhlia MA, Arvizu-Martínez J, Rodríguez-Romero J, Guerrero TD, Gutiérrez, F, Muhlia AA. 1994. Desarrollo científico y tecnológico del cultivo del robalo. Secretaría de Pesca. México. [Internet]/ Disponible en: https:// www.inapesca.gob.mx/portal/Publicaciones/Manuales/1994-Muhlia-Melo-etal.-Cultivo-de-robalo.pdf?download

25. Nayak SK. 2010. Role of gastrointestinal microbiota in fish. Aquac Res 41: 1553-1573. doi: 10.1111/j.1365-2109.2010.02546.x

26. Oulas A, Pavloudi C, Polymenakou P, Pavlopoulos GA, Papanikolaou N, Kotoulas G, Iliopoulos L. 2015. Metagenomics: tools and insights for analyzing next-generation sequencing data derived from biodiversity studies. Bioinform Biol Insights 9: 75-88. doi: 10.4137/BBI.S12462

27. Peso-Echarri P, Frontela-Saseta C, González-Bermúdez CA, Ros-Berruezo GF, Martínez-Graciá C. 2012. Polisacáridos de algas como ingredientes funcionales en acuicultura marina: alginato, carragenato y ulvano. Rev Biol Mar Oceanog 47: 373-381. doi: 10.4067/ S0718-355 19572012000300001

28. Pirarat N, Pinpimai K, Endo M, Katagiri T, Ponpornpisit A, Chansue $N$, Maita M. 2011. Modulation of intestinal morphology and immunity in nile 
tilapia (Oreochromis niloticus) by Lactobacillus rhamnosus GG. Res Vet Sci 91: e92-e97. doi: 10.1016/j.rvsc.2011.02.014

29. [PRODUCE] Ministerio de la Producción. 2017. Anuario estadístico pesquero y acuícola 2017. Lima, Perú. [Internet]. Disponible en: https://www.produce.gob.pe/documentos/estadisticas/anuarios/anuario-estadistico-pesca-2015.pdf

30. Ramírez C, Coronado J, Silva A, Romero J. 2018. Cetobacterium is a major component of the microbiome of giant Amazonian fish (Arapaima gigas) in Ecuador. Animals 8: 189. doi: 10.3390/ ani8110189

31. Ray AK, Ghosh K, Ringo E. 2012. Enzyme-producing bacteria isolated from fish gut: a review. Aquac Nutr 18: 465491. doi: 10.1111/j.1365-2095.2012.00943.x

32. Ramos Mogollon C, Palas Garcia L. 2013. Crecimiento, supervivencia y porcentaje de linfocitos en Centropomus sp (robalo) alimentado con tres dietas. Tesis de Ingeniero Pesquero. Tumbes: Univ. Nacional de Tumbes. $58 \mathrm{p}$.

33. Romero J, Feijoó CG, Navarrete P. 2012. Antibiotics in aquaculture - use, abuse and alternatives. In: Health and environment in aquaculture. InTech. doi: $10.5772 / 28157$
34. Sakai M, Yoshida T, Atsuta S, Kobayashi M. 1995. Enhancement of resistance to vibriosis in rainbow trout, Oncorhynchus mykiss (Walbaum), by oral administration of Clostridium butyricum bacterin. J Fish Dis 18: 187190. doi: 10.1111/j.1365-2761.1995.tb00276.x

35. Souza RM, Muriño JL, Vieira F, Buglione CC, Andreatta ER, Seiffert WQ, Cerqueira VR. 2010. Seleção de bactéria com potencial probiótico e utilização no cultivo de robalo-peva (Centropomus parallelus Poey, 1860). Bol Inst Pesca 36: 17-24.

36. Tarnecki AM, Burgos FA, Ray CL, Arias CR. 2017. Fish intestinal microbiome: diversity and symbiosis unraveled by metagenomics. J Appl Microbiol 123: 2-17. doi: $10.1111 /$ jam. 13415

37. Wang YB, Tian ZQ, Yao JT, Li WF. 2008. Effect of probiotics, Enteroccus faecium, on tilapia (Oreochromis niloticus) growth performance and immune response. Aquaculture 277: 203-207. doi:10.1016/j.aquaculture.2008.-03.007

38. Zorriehzahra MJ, Delshad ST, Adel M, Tiwari R, Karthik K, Dhama K, Lazado CC. 2016. Probiotics as beneficial microbes in aquaculture: an update on their multiple modes of action: a review. Vet Quart 36: 228-241. doi: $10.1080 / 01652176.2016 .1172132$ 